\title{
Many-Body Physics of Single and Double Spin-Flip Excitations in NiO
}

\author{
Abhishek Nag, ${ }^{1, *}$ H. C. Robarts, ${ }^{1,2}$ F. Wenzel, ${ }^{3}$ J. Li® ${ }^{1,4}$ Hebatalla Elnaggar, ${ }^{5}$ Ru-Pan Wang, ${ }^{5}$ A. C. Walters $\odot,{ }^{1}$ \\ M. García-Fernández $\odot,{ }^{1}$ F. M. F. de Groot, ${ }^{5}$ M. W. Haverkort, ${ }^{3}$ and Ke-Jin Zhou $\oplus^{1, \uparrow}$ \\ ${ }^{1}$ Diamond Light Source, Harwell Campus, Didcot OX11 ODE, United Kingdom \\ ${ }^{2}$ H. H. Wills Physics Laboratory, University of Bristol, Bristol BS8 1TL, United Kingdom \\ ${ }^{3}$ Institute for theoretical physics, Heidelberg University, Philosophenweg 19, 69120 Heidelberg, Germany \\ ${ }^{4}$ Beijing National Laboratory for Condensed Matter Physics, Institute of Physics, Chinese Academy of Sciences, Beijing 100190, China \\ ${ }^{5}$ Debye Institute for Nanomaterials Science, Utrecht University, Universiteitsweg 99, 3584 CG, Utrecht, The Netherlands
}

(Received 8 October 2019; accepted 16 January 2020; published 13 February 2020)

\begin{abstract}
Understanding many-body physics of elementary excitations has advanced our control over material properties. Here, we study spin-flip excitations in $\mathrm{NiO}$ using $\mathrm{Ni} L_{3}$-edge resonant inelastic x-ray scattering (RIXS) and present a strikingly different resonant energy behavior between single and double spin-flip excitations. Comparing our results with single-site full-multiplet ligand field theory calculations we find that the spectral weight of the double-magnon excitations originates primarily from the double spin-flip transition of the quadrupolar RIXS process within a single magnetic site. Quadrupolar spin-flip processes are among the least studied excitations, despite being important for multiferroic or spin-nematic materials due to their difficult detection. We identify intermediate state multiplets and intra-atomic core-valence exchange interactions as the key many-body factors determining the fate of such excitations. RIXS resonant energy dependence can act as a convincing proof of existence of nondipolar higher-ranked magnetic orders in systems for which, only theoretical predictions are available.
\end{abstract}

DOI: 10.1103/PhysRevLett.124.067202

Probing order parameters associated with various transitions are pivotal to systematic understanding of any materials' property. As we delve into more and more complex materials we find that the existing order parameters are coupled to each other and hence not easily separable. Many states also exist, whose order parameters completely evade characterization by conventional measurement techniques and hence dubbed as "hidden" [1-4]. Dynamical behavior of spin-dipolar excitations associated with long-range magnetic order for example, can be probed using inelastic neutron scattering (INS). However, higher rank spin-quadrupolar excitations at a magnetic site are inaccessible to such experiments [5]. These quadrupolar excitations, being time reversal invariant, may couple with electric dipoles giving rise to multiferroicity in materials or form long-range bound-magnon condensates in spinnematic systems, compelling their investigation $[4,6]$.

In the early days of development of resonant inelastic $\mathrm{x}$-ray scattering (RIXS), the possibility of detecting dipolar spin-flip excitations at $M, L$ absorption edges of transition metals was envisaged in the light of core-hole spin-orbit

Published by the American Physical Society under the terms of the Creative Commons Attribution 4.0 International license. Further distribution of this work must maintain attribution to the author(s) and the published article's title, journal citation, and DOI. coupling (SOC) in the intermediate state [7]. With continuous improvement in brilliance and energy resolution, successive experiments have established RIXS as a tool to unravel local or collective magnetic excitations [8-14]. Although spin-flip excitations involving two magnetic sites can be observed with various techniques [15-17], angular momentum conservation for the two-photon RIXS process uniquely allows it to even probe double spin-flip excitations at a single magnetic site, if $S>1 / 2$ [8,12-14]. Since noninteracting two-site spin-flip excitations cost an equal amount of energy as a single-site double spin-flip excitation, their past observations as inelastic features in RIXS spectra did not suffice to differentiate the two different processes [12-14]. While a single spin-flip excitation can be brought about by both dipolar and quadrupolar RIXS spin operators, a double spin-flip excitation can only result from the latter [18]. M. W. Haverkort in 2010 pioneered the idea that the cross section given by RIXS spin operators up to second rank can be described by fundamental $\mathrm{x}$-ray absorption (XAS) spectral functions, thereby implying a strong dependence of spectral weights of single and double spin-flip excitations on the incident photon energy around the absorption edges [18].

In this Letter, we provide the first experimental verification of this proposal and show the striking difference in the resonant behavior of single and double spin-flip excitations in archetypal $S=1$ antiferromagnetic insulator $\mathrm{NiO}$, by collecting RIXS spectra at different incident energies across 
the Ni $L_{3}$ edge. A minimal single-site full-multiplet simulation of RIXS spectral weights for the spin-flip processes is found to adequately describe the experimental results. These simulations demonstrate that single-site double spin-flip processes dominate the magnetic excitations with twice the single spin-flip energy-transfer in $\mathrm{NiO}$ when probed by RIXS. We further recognize Coulomb exchange interactions between core and valence electrons in the intermediate state to be instrumental in creating the single-site double spin-flip excitations, making them a many-body phenomena, unlike the case for dipolar excitations in $S=$ $1 / 2$ systems. Along with geometry and polarization, this Letter also serves to encourage experimentalists to identify and/or disentangle different excitations by the choice of incident photon energy.

$\mathrm{NiO}$ is an ideal material to initiate this kind of study since $\mathrm{Ni}^{2+}$ with nominally $3 d^{8}$ configuration and cubic symmetry has the lowest number of multiplet states for any $S>1 / 2$ system and the energetics associated with these states are well established [19,20]. It is also a strongly correlated antiferromagnetic insulator having an effective interatomic superexchange interaction $(\approx 110 \mathrm{meV})$ large enough to clearly separate the single $(\approx 110 \mathrm{meV})$ and double spin-flip excitations $(\approx 220 \mathrm{meV})$ with the energy resolution at Ni $L_{3}$-edge for RIXS experiments $(\approx 45 \mathrm{meV})$ $[12,14,21]$.

Figure 1(a) shows the scattering geometry used for the high-resolution ( $\triangle E=45 \mathrm{meV}$ ), Ni $L_{3}$-edge RIXS measurements done at the I21-RIXS beam line, Diamond Light Source, United Kingdom. A linear horizontally $(\pi)$ polarized x-ray beam was used, incident at an angle of $20^{\circ}$ with respect to the (100) surface plane of a polished $\mathrm{NiO}$ single crystal cooled down to $13 \mathrm{~K}$. The scattering angle was also kept fixed at $146^{\circ}$. The x-ray absorption spectrum (XAS) shown in Fig. 1(b) was measured using total electron yield in the same geometry, and RIXS spectra were collected for incident energies -3 to $+7 \mathrm{eV}$ with respect to the main resonance peak at $857.9 \mathrm{eV}$, in steps of $100 \mathrm{meV}$. The ground state in $\mathrm{NiO}$ is of spin-triplet ${ }^{3} A_{2 g}$ symmetry (parallel unpaired spins), and a typical RIXS spectrum shows excitations to both spin-singlet (antiparallel unpaired spins), and spin-triplet high-energy multiplet states of a $3 d^{8}$ ion, placed in an octahedral crystal field of $1.1 \mathrm{eV}$ influenced by a certain degree of interaction with $3 d^{9} \underline{L}$ and $3 d^{10} \underline{L}^{2}$ configurations due to hybridization with oxygen $p$ orbitals [20]. As such, the experimental RIXS map in Fig. 1(b) showing high energy-transfer inelastic features of $\mathrm{NiO}$ for different incident energies can be well simulated [Fig. 1(c)] by a single-site full-multiplet ligand field theory calculation implemented in the many-body code QUANTY [19,22] (see Sec. I of the Supplemental Material [23]). Spectral weights of excitations to the different multiplet states as marked in Fig. 1(c) are seen to be modulated with incident photon energy like previous reports [12,20,27,28]. What is unprecedented is the nearly 10 times increase in
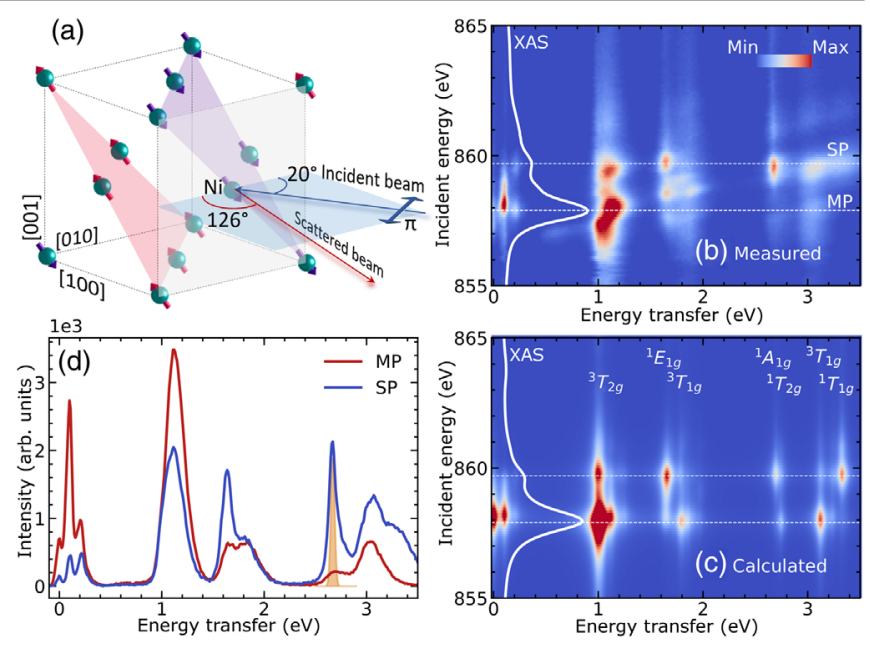

FIG. 1. All data presented in this work have been collected in the geometry shown in panel (a) [30]. Panel (b) shows the XAS collected on $\mathrm{NiO}$ along with the RIXS energy-transfer map upon changing incident energy. Simulation of the same map using single-site full multiplet ligand field theory implemented in QUANTY is shown in panel (c) marked with the RIXS final states for NiO. The main peak (MP) and satellite peak (SP) of the XAS are shown as dotted lines on the maps. Experimental RIXS incident energy line cuts at MP and SP of XAS in panel (b) are plotted separately in (d). A Gaussian line shape of instrumental resolution $(45 \mathrm{meV})$ is shaded at spin-singlet state excitation to ${ }^{1} A_{1 g}$ state for SP.

intensities of excitation to the spin-singlet multiplet state ${ }^{1} A_{1 g}$, as one goes from the main $\mathrm{Ni} L_{3}$ resonance peak (MP) to the satellite peak (SP) of XAS as shown in Fig. 1(d) [12]. Despite the strong SOC present in the core-hole $2 p^{5} 3 d^{9}$ configuration, there is a general consensus that the dominant multiplet states are spin-triplets at MP and spinsinglets at SP of XAS [19]. Since the final states of XAS act as the intermediate states of RIXS, the final states of RIXS are strongly affected by them. Therefore, the transition probability to spin-triplet final states (e.g., ${ }^{3} T_{2 g}$ ) is large compared to spin-singlet final states (e.g., ${ }^{1} A_{1 g}$ ) in RIXS, for incident photon energies around MP, while the opposite is true at SP as shown in Fig. 1(b). Excitation to the nondegenerate ${ }^{1} A_{1 g}$ state unaffected by SOC, from the ground spin-triplet ${ }^{3} A_{2 g}$ state involves only a single spinflipped orbital transition and therefore should be resolution limited [29]. The unfortunately similar energy-transfer values of ${ }^{1} A_{1 g}$ and ${ }^{1} T_{2 g}$ due to the level of crystal field splitting in $\mathrm{NiO}$ [20] however, do not allow us to resolve the two features. Even then, the peak is sharper than any orbital excitation observed in RIXS till date, as can be seen upon comparison with the shaded resolution function in Fig. 1(d).

In Fig. 1(d), besides high energy-transfer multiplet excitations, we see clearly two sharp low energy-transfer magnetic excitations $[12,14]$. We now turn to the discussion 
of incident photon energy dependence of these spin-flip excitations and the importance of many-body physics in the RIXS intermediate state to them as the main focus of the present work. The triply degenerate ${ }^{3} A_{2 g}$ ground state in the presence of interatomic superexchange molecular field can be viewed to be split according to the net spin orientation of the two unpaired $e_{g}$ electrons with respect to the field namely, $M_{s}=1,0$, or -1 . Apart from spindipolar excitations involving a single spin-flip at a magnetic site $\left(\Delta M_{s}=1\right)$, RIXS can also probe higher ranked quadrupolar spin excitations involving double spin-flips at a magnetic site $\left(\Delta M_{s}=2\right)[8,12]$. At the same time, like other probes, RIXS is also sensitive to quadrupolar spinexcitations involving simultaneous single spin-flips at multiple magnetic sites $\left(\Delta M_{s}=0\right)$.

The low energy-transfer RIXS map shown in Fig. 2(a), focuses on these pure spin-flip excitations in NiO. Sharp peaks are observed at an energy-transfer value of $0.105 \mathrm{eV}$ from the single-site single spin-flip excitations. After much speculation about their localized nature, these excitations have recently been demonstrated to have a collective behavior and deemed equivalent to magnons probed by INS [12-14]. Another set of excitations is observed at double this energy-transfer value, attributed to single or multiple-site double spin-flip excitations [12-14]. We refer to the single-site double spin-flip $\Delta M_{s}=2$ excitation as double-magnon [12], instead of bi- or two-magnon, which is often used for two-site double spin-flip $\Delta M_{s}=0$ excitation [15-17,31]. Trying to experimentally distinguish the double-magnons from noninteracting bimagnons therefore seems inconceivable. Neither a variation of scattering geometry helps, for both are expected to weakly disperse in

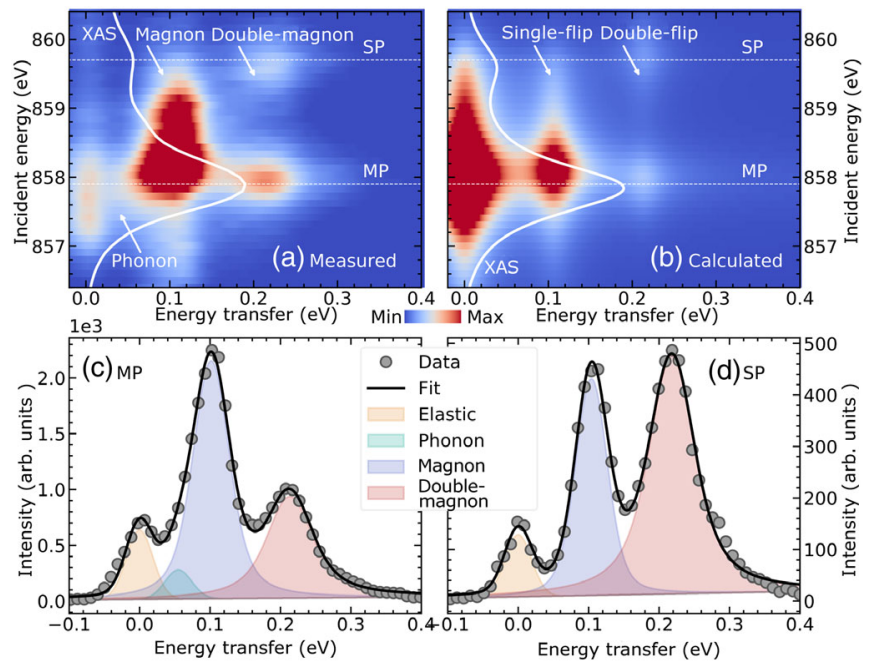

FIG. 2. Panels (a) and (b) show enlarged view of the low energy-transfer regions of the RIXS maps presented in Fig. 1(b) and (c) respectively, featuring the spin-flip excitations. Low energy-transfer RIXS line spectra at MP and SP are shown in panels (c) and (d) respectively, along with fits contributed by elastic, phonon, magnon, and double-magnon peaks. momentum space, nor a polarization analysis, since both are expected to be allowed for the non-spin-flip RIXS scattering channels [18,31,32]. A single-site full-multiplet calculation with an equivalent superexchange field as in $\mathrm{NiO}$ well reproduces also the low energy-transfer spin-flip excitations from the experiment [Fig. 2(b)]. Since NiO is known to have multiple magnetic domains at this temperature with smaller extents than the x-ray spot size, we have averaged over all possible contributions to simulate this map (see Fig. 1 of the Supplemental Material [23,33]). For a proper comparison with theory, we did a fitting of the low energy features, as the experimental spectra also contain phonon contributions which affects the apparent intensity around $0.1 \mathrm{eV}$. Two representative fits to the RIXS spectra at the MP and SP of XAS are shown in Figs. 2(c) and 2(d). While Gaussian line shapes were used to fit the elastic and phonon contributions, Gaussian convoluted Lorentzian line shapes were needed to fit the spin-flip excitations. The energy-transfer value of the magnon was kept as a free parameter and the best fits were obtained when the double spin-flip excitation energy-transfer was constrained to twice of this value. For both the spin excitations, the FWHM of the Gaussians were kept fixed as that of the elastic peak (45 meV), while the Lorentzian FWHM was allowed to vary. The fitted FWHM values of the Lorentzians for the double spin-flip excitations $(50 \mathrm{meV})$ are found to be larger than magnons $(25 \mathrm{meV})$ indicating their shorter lifetime and two-particle origin. Accordingly, they are only found around the Ni $L_{3}$ edge, while the magnons although much reduced in intensity, are observed far above and below the edge (see Fig. 3 of the Supplemental Material [23]). Any attempt to introduce additional peaks to account for further higher order excitations did not change the fitting results. Fits in this manner were done for all the incident energies (see Fig. 4 of
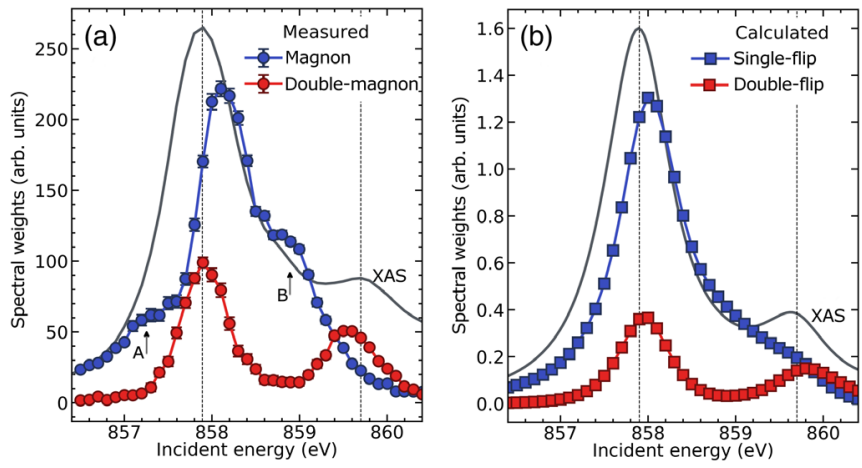

FIG. 3. Resonance behavior of the magnon and double-magnon excitations represented by their spectral weights extracted by fitting experimental RIXS line spectrum for each incident energy is plotted in panel (a) along with XAS. In panel (b) the spectral weights obtained from simulated RIXS spectra of single-site single and double spin-flip excitations and XAS in the same range of incident energies as in panel (a) is presented. The intensities of XAS data in both panels are scaled to compare with the spin-flip spectral weights. 


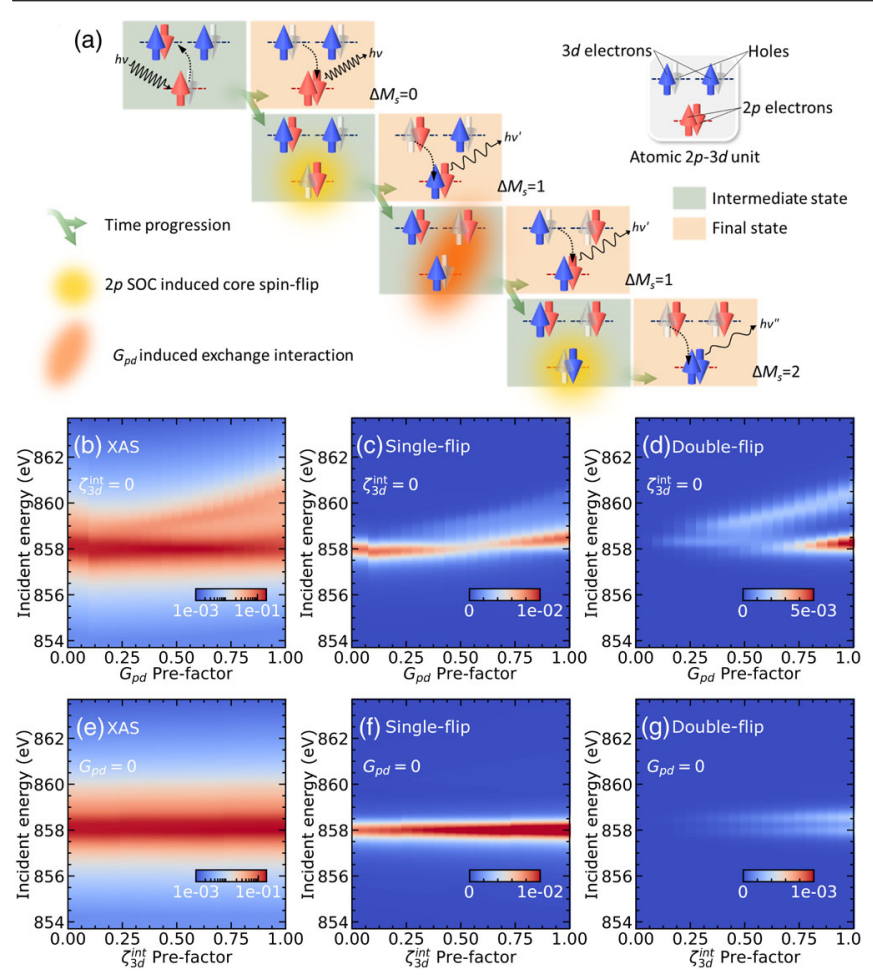

FIG. 4. Schematic representation of possible single-site spinflip states generated in a RIXS process in $d^{8}$ systems in presence of core-hole SOC and core-valence exchange Coulomb interaction $\left(G_{p d}\right)$ is shown in panel (a). For brevity, spin angular momentum change is shown for quantized spin projected along an axis. Distribution of intermediate states in RIXS, represented by XAS at the Ni $L_{3}$ edge are shown for varying strengths of $G_{p d}$ in panel (b). In panels (c) and (d), distribution and spectral weights of $\Delta M_{s}=1$ and $\Delta M_{s}=2$ spin-flip excitations are shown for each XAS spectrum given in panel (a). Similarly, effect of $\zeta_{3 d}^{\text {int }}$ on XAS spectra and $\Delta M_{s}=1$ and $\Delta M_{s}=2$ excitations across the $\mathrm{Ni}-L_{3}$ edge is presented in panels (e), (f), and (g), respectively. To isolate the effects of $G_{p d}$ and $\zeta_{3 d}^{\text {int }}$ on the excitations, while one is varied, the other is kept fixed at zero.

the Supplemental Material [23]), and the extracted spectral weights of the magnons and double spin-flip excitations at each energy are plotted in Fig. 3(a). For comparison, spectral weights from the single-site model calculation are shown in Fig. 3(b).

The resonant behaviors of the low energy magnetic excitations shown in Fig. 3(a) are strikingly different. The magnon excitation is seen to have maximum spectral weight at MP that gradually diminishes on reaching SP. For the double spin-flip excitations, when the incident photon energy is changed, we observe that the spectral weight peaks at both MP and SP with negligible presence in between. A similar trend is observed for the values extracted from the calculations done within a single-site full-multiplet framework in Fig. 3(b). Since the calculated spectral weights include only the single-site single and double spin-flip processes, their close resemblance to the resonant behaviors of the spectral weights of respective excitations from the experiment indicates that the feature observed at twice the magnon energy-transfer is predominantly from a single-site double spin-flip or a doublemagnon. In comparison, bimagnon excitations for this system scales with the complex isotropic spectral function $\sigma^{(0)}$ and hence a behavior different from experimental observations is expected (see Sec. VI of the Supplemental Material [23]). We observe enhanced spectral weights of magnon at 857.2 (A) and $858.8 \mathrm{eV}$ (B) when compared to the theory. Feature A is concomitant with incident photon energies having $\approx 0.5 \mathrm{eV}$ energy-transfer features in the RIXS map [Fig. 1(b)] and may have resulted from $\mathrm{NiO}_{5}$ surface states [34]. Feature B, absent from both the calculated magnon spectral weight and XAS, most likely originates from a small trigonal distortion present in the real sample (see Sec. VII of the Supplemental Material [23]).

To understand the importance of many-body physics in the intermediate states for spin-flip excitations in RIXS of $S=1$ systems, we schematically show these processes in Fig. 4(a). In the $2 p^{5} 3 d^{9}$ intermediate configuration, if only the $2 p^{5}$ core-hole SOC is considered, with spin-angular momentum of the $3 d^{9}$ valence electrons well-defined due to the absence of interaction between the core and valence electrons and $3 d$ SOC, the only possible transitions from this intermediate state involve $\Delta M_{s}=0$ and 1 , giving rise to only dipolar spin-flip excitations. In such a scenario, a $\Delta M_{s}=2$ excitation is not possible at all, since one unit of photon orbital angular momentum is already transferred to create the intermediate state. However in reality, the valence-electrons strongly couple to the core-electrons in the intermediate state via exchange Coulomb interactions [35]. Therefore for both the core and valence orbitals spinangular momentum is no longer a good quantum number, leading to $\Delta M_{s}=0,1$, and 2 excitations. To check the validity of this argument, we did model calculations for a $\mathrm{Ni}^{2+}$ ion in $O_{h}$ symmetry (see Sec. VIII of the Supplemental Material [23]). All parameters are kept the same as in $\mathrm{NiO}$, with the only difference being the intermediate state SOC of $3 d^{9}$ level ( $\zeta_{3 d}^{\text {int }}$ ) set to zero while we varied the strength of intra-atomic core-valence Coulomb exchange interaction $\left(G_{p d}\right)$ and the results are shown in Figs. 4(b)-4(d). One can clearly see that in the absence of $G_{p d}$, although $\Delta M_{s}=1$ excitations are observed, there are no $\Delta M_{s}=2$ excitations [Figs. 4(c) and 4(d)]. The $\Delta M_{s}=2$ excitations begin to appear and continue to grow in intensity only after the introduction of $G_{p d} . G_{p d}$ is known to strongly modify relative positions of XAS final states as seen in Fig. 4(b) [36], thereby also dictating the resonance behavior of $\Delta M_{s}=1,2$ excitations. $F_{p d}$, the direct Coulomb interaction between the core and the valence electrons, is not found to generate $\Delta M_{s}=$ 2 spin-flips on its own, but affects their relative intensities and resonance in presence of $G_{p d}$ (see Fig. 7 of the Supplemental Material [23]). Another parameter that can 
affect the spin-angular momentum of the $3 d^{9}$ electrons in the intermediate state is the $\mathrm{SOC} \zeta_{3 d}^{\text {int }}$. We find that influence of $\zeta_{3 d}^{\text {int }}$ is much smaller than $G_{p d}$ for the generation of $\Delta M_{s}=2$ excitations [Fig. 4(g)]. Ground state $3 d$ SOC has no effect on the excitations in $\mathrm{NiO}$ since $M_{L}=0$. It should be noted that in $S=1 / 2$ cuprates, neither the core-valence interactions nor the valence level SOC is present in the intermediate state to affect the magnetic excitations in $\mathrm{Cu}$ $L_{3}$-edge RIXS. For indirect RIXS at $\mathrm{Cu} K$ edge, effective core-hole screening has also been shown to selectively enhance magnetic excitations [37].

We have demonstrated how many-body interactions in the intermediate state affect the spectral weight distribution of spin-flip magnetic RIXS processes in $3 d^{8} S=1$ systems. The exchange core-valence Coulomb interaction and valence SOC in the intermediate state are identified as the key factors behind the origin of the single-site double spinflip quadrupolar excitations. Comparing results of RIXS experiments with single-site full-multiplet calculations, we show that double spin-flip single-site quadrupolar processes predominate excitations that have double the energy of magnons in NiO. Unlike $S=1 / 2$ systems where in the intermediate state Heisenberg exchange interactions with neighboring sites are completely blocked, here the perturbations giving rise to bimagnon excitations are weaker. We here provide a clear experimental method to decide which type of excitation one measures, like local double flip $\left(\Delta M_{s}=2\right)$ or nonlocal double-flip $\left(\Delta M_{s}=0\right)$, without the need to know the full theory of propagating magnon. RIXS being the only existing technique at present that can probe the dynamics of these quadrupolar excitations, our observations showing fundamentally different resonance behavior of dipolar and quadrupolar excitations can be used to identify excitations in magnetically frustrated systems that are theoretically predicted to have only higher rank magnetic multipoles and a suppressed long-range dipolar order.

We acknowledge Diamond Light Source for beam time on I21-RIXS beam line. We thank Thomas Rice for his help during the RIXS measurements. We would also like to thank the Materials Characterisation Laboratory team for help on the Laue instrument in the Materials Characterisation Laboratory at the ISIS Neutron and Muon Source.

*abhishek.nag@diamond.ac.uk

†ejin.zhou@diamond.ac.uk

[1] L. Savary and T. Senthil, arXiv:1506.04752.

[2] H.-H. Kung, S. Ran, N. Kanchanavatee, V. Krapivin, A. Lee, J. A. Mydosh, K. Haule, M. B. Maple, and G. Blumberg, Phys. Rev. Lett. 117, 227601 (2016).

[3] A. S. Patri, A. Sakai, S. Lee, A. Paramekanti, S. Nakatsuji, and Y. B. Kim, Nat. Commun. 10, 4092 (2019).
[4] Y. Kohama, H. Ishikawa, A. Matsuo, K. Kindo, N. Shannon, and Z. Hiroi, Proc. Natl. Acad. Sci. U.S.A. 116, 10686 (2019).

[5] Y.-J. Kim, A. P. Sorini, C. Stock, T. G. Perring, J. van den Brink, and T. P. Devereaux, Phys. Rev. B 84, 085132 (2011).

[6] M. Akaki, D. Yoshizawa, A. Okutani, T. Kida, J. Romhányi, K. Penc, and M. Hagiwara, Phys. Rev. B 96, 214406 (2017).

[7] F. M. F. de Groot, P. Kuiper, and G. A. Sawatzky, Phys. Rev. B 57, 14584 (1998).

[8] L. J. P. Ament, M. van Veenendaal, T. P. Devereaux, J. P. Hill, and J. van den Brink, Rev. Mod. Phys. 83, 705 (2011).

[9] L. Braicovich et al., Phys. Rev. Lett. 104, 077002 (2010).

[10] J. Schlappa et al., Nature (London) 485, 82 (2012).

[11] S. G. Chiuzbăian, G. Ghiringhelli, C. Dallera, M. Grioni, P. Amann, X. Wang, L. Braicovich, and L. Patthey, Phys. Rev. Lett. 95, 197402 (2005).

[12] G. Ghiringhelli, A. Piazzalunga, C. Dallera, T. Schmitt, V. N. Strocov, J. Schlappa, L. Patthey, X. Wang, H. Berger, and M. Grioni, Phys. Rev. Lett. 102, 027401 (2009).

[13] G. Fabbris et al., Phys. Rev. Lett. 118, 156402 (2017).

[14] D. Betto, Y. Y. Peng, S. B. Porter, G. Berti, A. Calloni, G. Ghiringhelli, and N. B. Brookes, Phys. Rev. B 96, 020409(R) (2017).

[15] T. P. Devereaux and R. Hackl, Rev. Mod. Phys. 79, 175 (2007).

[16] L. Chaix et al., Phys. Rev. B 97, 155144 (2018).

[17] T. Huberman, R. Coldea, R. A. Cowley, D. A. Tennant, R. L. Leheny, R. J. Christianson, and C. D. Frost, Phys. Rev. B 72, 014413 (2005).

[18] M. W. Haverkort, Phys. Rev. Lett. 105, 167404 (2010).

[19] M. W. Haverkort, M. Zwierzycki, and O. K. Andersen, Phys. Rev. B 85, 165113 (2012).

[20] G. Ghiringhelli, M. Matsubara, C. Dallera, F. Fracassi, R. Gusmeroli, A. Piazzalunga, A. Tagliaferri, N. B. Brookes, A. Kotani, and L. Braichovich, J. Phys. Condens. Matter 17, 5397 (2005).

[21] M. T. Hutchings and E. J. Samuelson, Phys. Rev. B 6, 3447 (1972).

[22] M. W. Haverkort, J. Phys. Conf. Ser. 712, 012001 (2016).

[23] See Supplemental Material at http://link.aps.org/ supplemental/10.1103/PhysRevLett.124.067202, which includes Refs. [24-26] along with details of the experiments and theory.

[24] L. C. Bartel and B. Morosin, Phys. Rev. B 3, 1039 (1971).

[25] B. Fromme, d-d Excitations in Transition Metal Oxides (Springer Verlag, Berlin, 2001), Vol. 170, p. 22.

[26] M. Minola et al., Phys. Rev. Lett. 114, 217003 (2015).

[27] H. Ishii, Y. Ishiwata, R. Eguchi, Y. Harada, M. Watanabe, A. Chainani, and S. Shin, J. Phys. Soc. Jpn. 70, 1813 (2001).

[28] M. Magnuson, S. M. Butorin, J.-H. Guo, and J. Nordgren, Phys. Rev. B 65, 205106 (2002).

[29] R. Rückamp, E. Benckiser, M. W. Haverkort, H. Roth, T. Lorenz, A. Freimuth, L. Jongen, A. Möller, G. Meyer, and P. Reutler, New J. Phys. 7, 144 (2005).

[30] K. Momma and F. Izumi, J. Appl. Crystallogr. 44, 1272 (2011).

[31] V. Bisogni et al., Phys. Rev. B 85, 214527 (2012).

[32] R. Fumagalli et al., Phys. Rev. B 99, 134517 (2019). 
[33] I. Sänger, V. V. Pavlov, M. Bayer, and M. Fiebig, Phys. Rev. B 74, 144401 (2006).

[34] K. Satitkovitchai, Y. Pavlyukh, and W. Hübner, Phys. Rev. B 67, 165413 (2003).

[35] F. M. F. de Groot, Coord. Chem. Rev. 249, 31 (2005).
[36] M. van Veenendaal, J. B. Goedkoop, and B. T. Thole, Phys. Rev. Lett. 77, 1508 (1996).

[37] C. J. Jia, C.-C. Chen, A. P. Sorini, B. Moritz, and T. P. Devereaux, New J. Phys. 14, 113038 (2012). 